\title{
MODELAGEM E SIMULAÇÃO DE LAVADOR VENTURI UTILIZANDO FLUIDODINÂMICA COMPUTACIONAL
}

\author{
A. A. ZERWAS $^{1 *}$, J. L. DE PAIVA ${ }^{1}$ \\ ${ }^{1}$ Escola Politécnica da Universidade de São Paulo, Departamento de Engenharia Química \\ e-mail: alexander.zerwas@usp.com
}

\begin{abstract}
RESUMO
Lavadores Venturi são utilizados industrialmente para o controle da emissão de poluentes. Simulou-se a variação de vazões de líquido e gás em lavador Venturi industrial utilizando técnicas de CFD (Fluidodinâmica Computacional). Com o auxílio do software ANSYS CFX 15.0, simulou-se o escoamento bifásico, utilizando o modelo de fluidos interpenetrantes (abordagem Euler-Euler) e o modelo de turbulência $k-\varepsilon$ padrão. Observou-se que a variação de pressão ao longo do Venturi calculada é, de forma geral, muito próxima da prevista pelos modelos clássicos de Boll (1973) e Viswanathan (1985). No entanto, para algumas condições de vazão de gás e líquido, observaram-se diferenças entre os resultados obtidos por simulação e os previstos pelos modelos clássicos. Para a geometria utilizada do lavador, notou-se a presença de regiões de recirculação para as condições estudadas, possivelmente explicando as diferenças entre as simulações e os modelos. Obtiveram-se, também, resultados da distribuição de líquido ao longo do lavador.
\end{abstract}

\section{INTRODUÇÃO}

$\mathrm{Na}$ indústria, diversos equipamentos de lavagem de gases são utilizados para remoção de poluentes de correntes gasosas e assim, atender aos limies de emissão definidos pelas agências regulamentadoras (CONAMA). Dentre esses equipamentos, o lavador de gás Venturi por via úmida apresenta uma alta eficiência de remoção de poluentes, construção e implementação simples, de acordo com Ahmadvand e Talaie (2010).

O lavador Venturi é geralmente composto por 3 partes, sendo uma região onde ocorre a convergência do gás a ser lavado (região convergente); uma região de secção transversal constante (garganta); e uma secção divergente (região divergente).

$\mathrm{O}$ funcionamento do equipamento ocorre pela inserção do líquido de lavagem (geralmente água) na forma de filme ou jato na corrente de gás com poluente. Devido à alta tensão cisalhante proporcionada pelo escoamento do gás, o líquido é dispersado em gotas de diversos tamanhos, as quais são transportadas por esse ao longo do Venturi.

Diversos autores modelaram a queda de pressão no lavador Venturi utilizando abordagens diferentes. Boll (1973) modelou a queda de pressão assumindo quebra imediata do jato de líquido em gotículas de tamanho uniforme e dispersão homogênea desse na secção tranversal do lavador, perda de carga devido ao atrito (proporcional à razão de líquido por gás) e efeito da geometria. $\mathrm{O}$ modelo pode ser expresso pela Equação 1.

$$
-\frac{d P}{\rho_{g}}=v_{g} d v_{g}+m v_{g} d v_{d}+\frac{(m+1) f v_{g}^{2}}{2 D_{h}} d x
$$


Em 1977, Yung, Barbarika e Calvert (YUNG; BARBARIKA; CALVERT, 1977) propuseram um modelo simplificado de queda de pressão, assumindo que a troca de momento do gás com o líquido ocorria apenas devido ao arrasto das gotas.

Em 1985, Leith, Cooper e Rudnick (LEITH; COOPER; RUDNICK, 1985) propuseram uma melhoria ao modelo de Yung, Barbarika e Calvert (1977), considerando a recuperação de pressão na região divergente do lavador. Neste mesmo ano, Vishwanathan (1985) utilizou a teoria de escoamento anular para a modelagem de lavadores Venturi, considerando que o líquido inserido não era totalmente atomizado, sendo que parte desse escoava por uma película na parede do lavador, conforme visto na Equação 2.

$$
\begin{aligned}
-\frac{d P}{\rho_{g}} & =\left[\alpha_{g} a_{c} v_{g} d v_{g}\right]+\left[m C \alpha_{g} a_{c} v_{g} d v_{d}\right] \\
& +\left[m(1-C) \alpha_{g} a_{c} v_{g} d v_{f}\right]+\left[\phi_{c}^{2} \frac{f M_{c}^{2}}{2 D_{h} \rho_{g} \rho_{c} A_{T}^{2}} d x\right]
\end{aligned}
$$

Gonçalves et al. (2001) compararam modelos propostos de queda de pressão de vários autores com dados experimentais obtidos com lavadores de diferentes geometrias.

Com os avanços computacionais, a utilização e resolução das equações de transporte em três dimensões se tornou possível em sistemas multifásicos. Em 2006, Pak e Chang (PAK; CHANG, 2006) realizaram um estudo utilizando CFD (Fluido dinâmica Computacional) para a obtenção da perda de pressão ao longo do lavador Venturi e a coleta de partículas de poluentes pelas gotas de líquido, utilizando para tanto, a abordagem Euler-Lagrange para a resolução do escoamento multifásico. Guerra et al. (2012) utilizaram a abordagem Euler-Euler com o método de volume de fluido para a resolução do escoamento de líquido e gás. Os autores utilizaram o modelo de turbulência $k-\varepsilon$ padrão e o software ANSYS FLUENT e compararam os resultados obtidos pelas simulações com dados experimentais, obtendo boas adequações.

Majid et al. (2013) simularam a quebra do jato de líquido no lavador Venturi utilizando o modelo de quebra CAB, implementado no software comercial ANSYS CFX. Os autores utilizaram o modelo de turbulência RNG $k-\varepsilon$ e a abordagem EulerLagrange para a resolução do escoamento multifásico, comparando os resultados obtidos com dados experimentais.

\section{MATERIAIS E MÉTODOS}

\subsection{Equipamento de Lavagem}

$\mathrm{O}$ equipamento de lavagem industrial utilizado nas simulações discutidas no presente trabalho é constituído de um lavador Venturi acoplado à uma coluna de absorção, de modo que o gás lavado pelo Venturi contendo gotículas de líquido e poluentes seja novamente lavado na coluna, aumentando-se assim o diâmetro destas gotas por processos de coalescência e promovendo o processo de separação do líquido da fase gás, que ocorre por sedimentação.

Devido a complexidade de se realizar simulações numéricas de duas fases com a geometria completa (Venturi + tanque de lavagem), optou-se por se modelar apenas o lavador Venturi, cujo esquema simplificado é mostrado na Figura 1 e as principais dimensões especificados na Tabela 1.

A alimentação de líquido de lavagem no Venturi ocorre por meio de um bico injetor de cone cheio na direção do escoamento do gás, localizado no centro do tubo do lavador e a $400 \mathrm{~mm}$ da entrada, ou seja, no começo da secção convergente. 
Tabela 1 - Especificações do lavador Venturi

Especificação

Dimensão $(\mathrm{mm})$

Diâmetro (D) 600

Diâmetro garganta $\left(\mathrm{D}_{\mathrm{g}}\right)$

Comprimento da entrada $\left(l_{\mathrm{e}}\right)$

Comprimento da secção convergente $\left(l_{c}\right)$

Comprimento da garganta $\left(l_{\mathrm{g}}\right)$

Comprimento da secção divergente $\left(l_{d}\right)$

Comprimento da saìda $\left(1_{\mathrm{s}}\right)$

Figura 1 - Esquema representativo do lavador Venturi

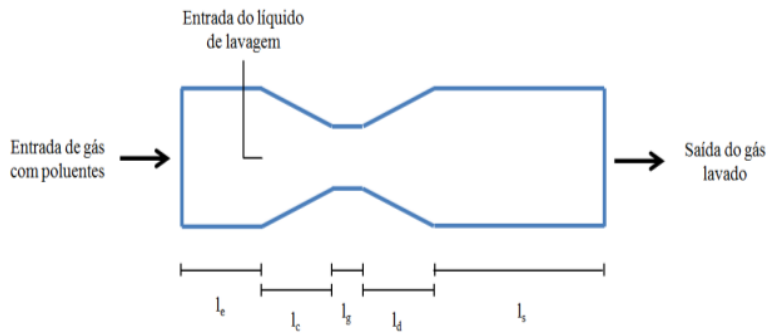

\subsubsection{Condições de Operação}

Os dados de operação do lavador foram obtidos com base em um lavador industrial, sendo a corrente gasosa com vazão de 15100 $\mathrm{m}^{3} / \mathrm{h}$, em pressão atmosférica e temperatura de $25{ }^{\circ} \mathrm{C}$, e a vazão de líquido de lavagem de $18 \mathrm{~m}^{3} / \mathrm{h}$, a $25^{\circ} \mathrm{C}$. Devido a presença de um sistema de pré-lavagem do gás localizado à montante do lavador Venturi, considera-se que o gás troca calor e massa com o líquido da pré-lavagem, entrando esse no lavador a uma temperatura próxima a ambiente, considerada como sendo de $25^{\circ} \mathrm{C}$, e com umidade relativa próxima a da saturação.
Desse modo, pode-se supor que no lavador Venturi, as gotas de líquido injetadas não reduzirão de tamanho devido aos processos de transferência de calor e massa.

\subsubsection{Diâmetro de gota}

Como não se dispõe de dados do diâmetro médio das gotas de líquido geradas pelo bico injetor utilizado, optou-se por usar a correlação proposta por Boll et al. (1974), conforme a Equação 3 e utilizada em outros trabalhos da literatura (PAK; CHANG, 2006; AHMADVAND; TALAIE, 2010; RAHIMI; NIKSIAR; MOBASHERI, 2011).

$$
D_{32}=\frac{4,22 * 10^{-2}+5,77 * 10^{-3}\left(\frac{1000 L}{G}\right)^{1,922}}{v_{g g}^{1,602}}
$$

Utilizando os dados de operação do sistema de lavagem, a geometria do Venturi e a Equação 3, obteve-se para o diâmetro de Sauter das gotas geradas o valor de 230 microns.

\subsection{Simulação Computacional}

Construiu-se a geometria do equipamento mostrada na Figura 1, porém aumentou-se a região $1_{\mathrm{s}}$ de $0,35 \mathrm{~m}$ para $2,2 \mathrm{~m}$, para efeito das condições de contorno na saída.

O sistema de coordenadas utilizado nos resultados foi posicionado na intersecção do eixo de simetria do equipamento com o plano da secção de entrada. A geometria construída está ilustrada nas Figuras 2 e 3 . O escoamento do gás ocorre na direção e sentido crescente do eixo $\mathrm{Y}$.

Figura 2 - Geometria do lavador Venturi (vista lateral)

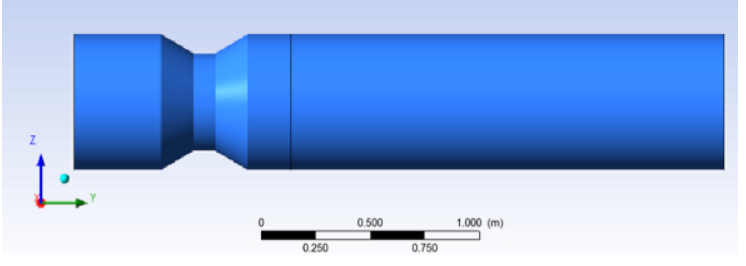


Figura 3 - Geometria do lavador Venturi (vista frontal)

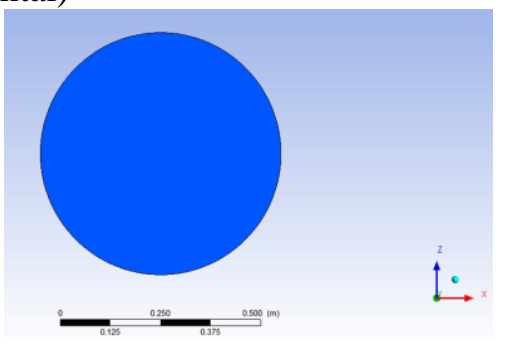

De modo a simular o escoamento bifásico no lavador Venturi, utilizou-se o software comercial ANSYS CFX 15.0, considerando-se o escoamento incompressível e isotérmico. A abordagem Euler-Euler foi utilizada para a resolução do escoamento bifásico. Esta abordagem modela os fluidos como interpenetrantes, assumindo a presença de frações volumétricas das fases contínua (gás) e dispersa (líquido) nas equações da continuidade e de momento conforme expresso, respectivamente, nas Equações 4 e 5.

$\frac{\partial\left(X_{a} \rho_{a}\right)}{\partial t}+\nabla \cdot\left(X_{a}\left(\rho_{a} \overrightarrow{u_{a}}\right)\right)=S_{a}$

$\frac{\partial\left(X_{a} \rho_{a} \overrightarrow{u_{a}}\right)}{\partial t}+\nabla_{1}\left(X_{a}\left(\rho_{a} \overrightarrow{u_{a} u_{a}}\right)\right)=-X_{a} \nabla P_{a}+\nabla_{\cdot}\left(X_{a} \mu_{a}\left(\nabla \overrightarrow{u_{a}}+\left(\nabla \overrightarrow{u_{a}}\right)\right)+\overrightarrow{M_{a b}}\right.$

O termo de produção da equação de continuidade $\left(S_{a}\right)$ foi utilizado para se modelar a entrada de líquido no lavador, na região do bico injetor. $\mathrm{O}$ termo $\left(M_{a b}\right)$ na Equação 5 representa a troca interfacial de momento entre as duas fases e supôs-se que este ocorre apenas devido a força de arraste sofrida pela fase dispersa na fase contínua. $\mathrm{O}$ termo de troca de momento é então dado conforme Equação 6.

$\overrightarrow{M_{a b}}=\frac{3}{4} \frac{C_{D}}{d} X_{b} \rho_{a}\left|\overrightarrow{u_{b}}-\overrightarrow{u_{a}}\right|\left(\overrightarrow{u_{b}}-\overrightarrow{u_{a}}\right)$
Nas simulações realizadas, considerouse que a velocidade de injeção da fase dispersa é nula.

O coeficiente de arrasto $\left(C_{D}\right)$ utilizado é dado pela correlação de Schiller-Naumann, conforme Equação 7 (ANSYS, 2013). No caso do número de Reynolds ser superior a $10^{4}$, considera-se o valor de 0,44 para o coeficiente.

$C_{D}=\frac{24}{R e}\left(1+0,15 R e^{0,687}\right)$

Para o equacionamento do escoamento turbulento empregou-se o modelo $k$ - $\varepsilon$ padrão com lei de parede automática. O efeito da gravidade foi considerado nas simulações.

\subsubsection{Malha utilizada}

A malha utilizada nas simulações foi criada usando o software de geração de malha disponível no pacote comercial ANSYS 15.0 ("ANSYS Meshing"). Buscou-se a criação de uma malha estruturada ao invés de uma tetraédrica, visando-se assim a diminuição do número de elementos necessários.

Além disso, criou-se perto das paredes do equipamento uma região com malha estruturada de altura controlável. Ajustes nesta altura foram feitos de modo que o critério de Yplus (para a condição de velocidade de operação) fosse atendido para o modelo de turbulência utilizado. Para a condição de operação descrita no Item 2.1.1, o valor máximo de Yplus foi de 230, na região da garganta e o valor mínimo de 10 , com um valor médio de 98 ao longo da geometria.

A mesma malha foi utilizada nas simulações com diferentes vazões de gás e líquido, possuindo cerca de 490.000 elementos, conforme pode ser visto na Figura 4. 
Figura 4 - Malha utilizada nas simulações

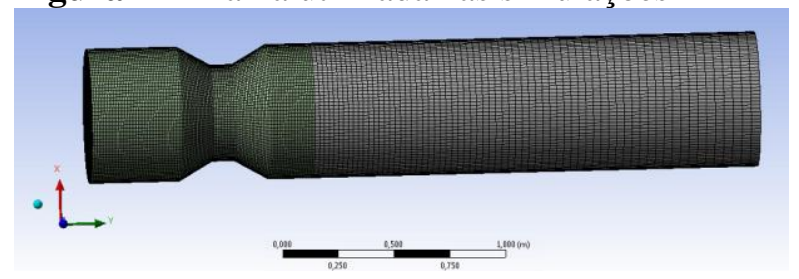

As Figuras 5 e 6 mostram as malhas nos cortes realizados no centro do lavador e na região da garganta, respectivamente. Nota-se a presença da malha com altura definida, conforme explicado anteriormente.

Figura 5 - Malha em secção no centro do lavador

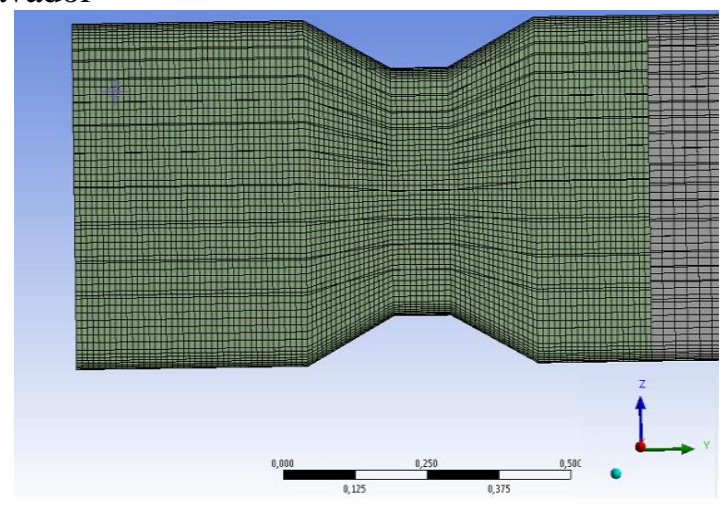

Figura 6 - Malha em secção no meio da região da garganta

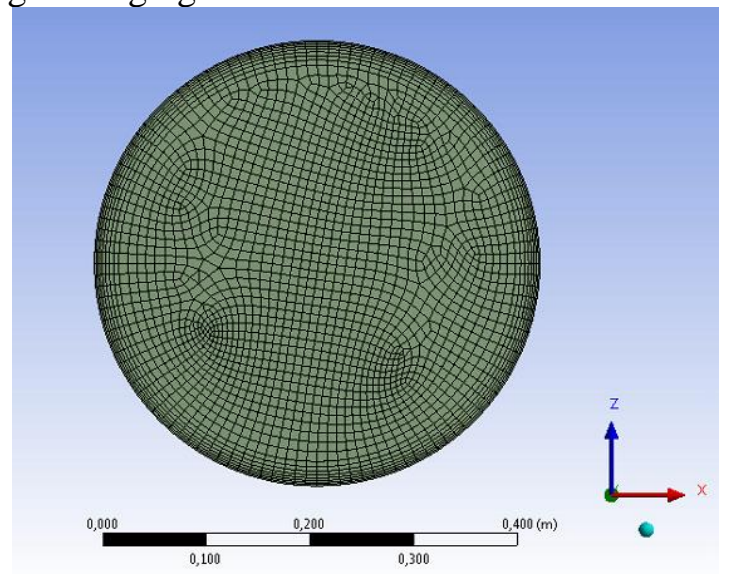

\subsubsection{Condições Estudadas}

Simularam-se nove condições diferentes de operação do lavador Venturi em torno do valor típico de operação, conforme pode ser visto na Tabela 2, combinando-se os valores de vazão de líquido com a vazão da fase gás.

Tabela 2 - Condições de vazão de líquido e gás simuladas

\begin{tabular}{lr}
\hline Especificação & Vazão $\left(\mathrm{m}^{3} / \mathrm{h}\right)$ \\
\hline & 9 \\
Vazão de líquido & 18 \\
& 21,6 \\
& 12080 \\
Vazão de gás & 15100 \\
& 18120
\end{tabular}

\subsubsection{Critério de Convergência Utilizado}

Para a convergência das simulações, primeiro se rodou no simulador o escoamento apenas da fase gás em estado estacionário, utilizando resolução UPWIND, parâmetro de relaxação de 0,6 e com critério de convergência (RMS) de $10^{-5}$. Após a convergência da fase gás, utilizaram-se os dados gerados e inseriu-se a fase líquida como termo fonte na posição indicada pelo Item 2.1, iniciando-se, assim, uma nova simulação considerando-se as duas fases em estado transiente. Definiu-se um intervalo de passo de $0,02 \mathrm{~s}$ e 10 iterações para cada passo (apenas na simulação com o maior valor de vazão de líquido e de gás usou-se 3 iterações por passo), com um tempo total de simulação de $15 \mathrm{~s}$. A convergência (RMS) para o estado transiente foi mantida em $10^{-5}$.

\subsubsection{Avaliação da Convergência}

De modo a se verificar a convergência do estado transiente do Item 2.2.3, utilizaramse dois pontos de referência, localizados no centro do lavador e posicionados a $0,6 \mathrm{~m}$ e a $1,1 \mathrm{~m}$ do sistema de coordenadas, na direção do escoamento do gás. Esses pontos foram usados para se monitorar os valores da pressão, velocidades da fase contínua nas três direções e a fração volumétrica da fase líquida. 
Com o decorrer da simulação no estado transiente, se observou a variação dos valores dessas propriedades e quando essas se estabilizavam, admitia-se que o estado estacionário havia sido atingido. Os dados utilizados nas análises foram todos coletados na última iteração de cada simulação, ou seja, quando essas atingiam $15 \mathrm{~s}$. Todas as simualções realizadas atingiram o estado estacionário pelo critério estabelecido.

\subsection{Levantamento de Dados das Simulações}

Após se analisar a convergência de cada simulação pelo critério descrito no Item 2.2.4, criaram-se 22 planos perpendiculares ao escoamento do gás no lavador para a coleta dos valores médios da pressão. Definiram-se a colocação de 5 planos, igualmente espaçados, em cada uma das regiões: convergente, garganta e divergente; além de 7 outros planos na região da saída. A distância na coordenada $\mathrm{Y}$ dos planos de transição de região ao sistema de coordenadas definido pode ser visto na Tabela 3.

Tabela 3 - Distância $\mathrm{Y}$ ao sistema de coordenadas

\begin{tabular}{cc}
\hline Plano & $\begin{array}{c}\text { Distância } \\
(\mathrm{mm})\end{array}$ \\
\hline
\end{tabular}

Plano 1 - entrada de líquido no lavador Venturi

Plano 6 - fronteira da região convergente e garganta

Plano 11 - fronteira da região da garganta e divergente

Plano 16 - fronteira da região divergente e saída

Plano 21 - último plano espaçado uniformemente

Plano 22 - final da geometria
Para se obter a pressão média em cada plano, utilizou-se uma ferramenta do software que ponderou o valor da pressão pela área.

\section{RESULTADOS E DISCUSSÃO}

As simulações realizadas foram comparadas com os modelos de Boll (1973), dado pela Equação 1, e de Viswanathan (1985), dado pela Equação 2, utilizando as vazões de líquido e de gás referentes a cada simulação e com diâmetro de gota igual para todas (Item 2.1.2). O coeficiente de atrito usado nos modelos foi calculado supondo as paredes do lavador como totalmente lisas e a velocidade das gotas na direção do escoamento do gás como nulas.

A Figura 7 mostra o comparativo entre as simulações realizadas com CFD e o modelo de Boll (1973), para uma vazão de líquido constante e diferentes vazões de gás $\left(\mathrm{Q}-\mathrm{m}^{3} / \mathrm{h}\right)$. Pode-se perceber que o modelo e as simulações representam o mesmo comportamento para a perda de pressão na região convergente e da garganta do lavador, porém para a maior vazão de gás, o comportamento do modelo difere da simulação na região da secção divergente. Nota-se que para um comprimento da secção de saída de 0,4 m, os valores de perda de pressão são próximos, sendo superestimados pelo modelo nas vazões de gás menores e subestimado para a vazão maior. 
Figura 7 - Influência da vazão do gás para injeção de líquido constante $\left(\mathrm{L}-18 \mathrm{~m}^{3} / \mathrm{h}\right)$, para o modelo de Boll (1973)

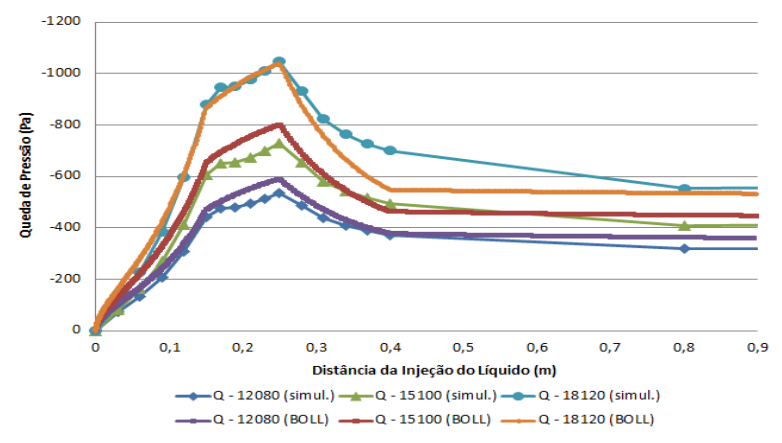

As Figuras 8 e 9 comparam os valores de queda de pressão no lavador obtidos pelos cálculos dos dois modelos teóricos, com as simulações realizadas para diferentes vazões de líquido $\left(\mathrm{L}-\mathrm{m}^{3} / \mathrm{h}\right)$ e vazão de gás fixa. Percebe-se que o modelo de Viswanathan (1985) se adequa melhor aos dados obtidos pelas simulações na região da secção convergente e garganta; para a secção divergente apenas para os casos com vazão de líquido maiores. Para o caso da vazão de líquido mais baixa, nota-se que ambos os modelos não foram bem representados pela simulação na região da secção divergente do lavador.

As perdas de pressão total no equipamento, para um comprimento de $0,4 \mathrm{~m}$ da secção de saída, foram próximas aos obtidos nas simulações em comparação com o modelo de Viswanathan (1985), o qual superestimou no caso das duas vazões de líquido maiores e subestimando no caso da vazão menor.
Figura 8 - Influência da vazão de líquido para injeção de gás constante $(\mathrm{G}-12080$ $\mathrm{m}^{3} / \mathrm{h}$ ), para o modelo de Boll (1973)

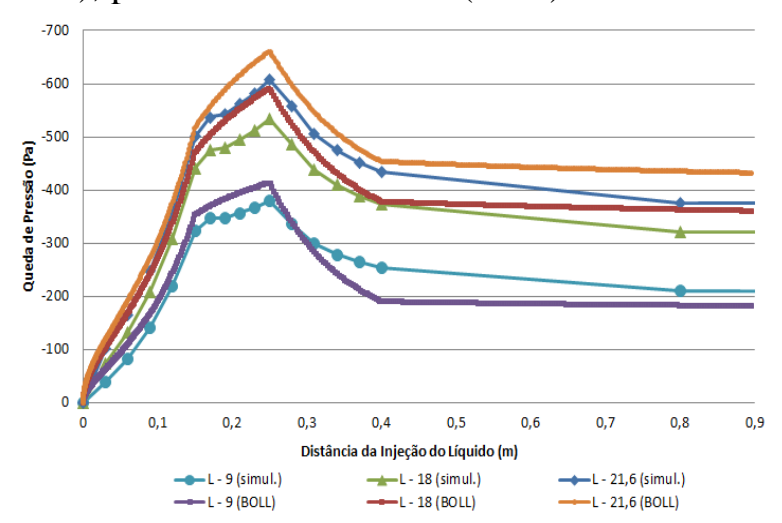

Figura 9 - Influência da vazão de líquido para injeção de gás constante $(\mathrm{G}-12080$ $\mathrm{m}^{3} / \mathrm{h}$ ), para o modelo de Viswathan (1985)

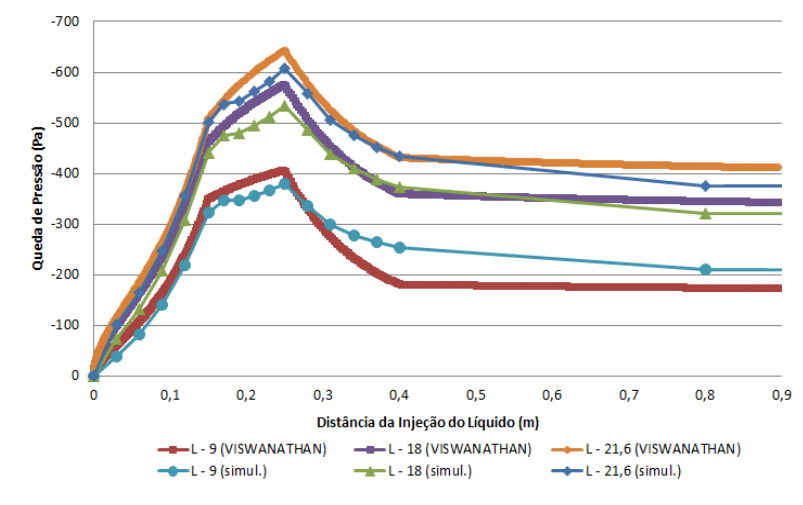

Comparando-se os dados de queda de pressão para a maior vazão de gás do modelo de Viswanathan (1985) com as simulações realizadas, pode-se perceber pela Figura 10, que a adequação ocorre para a região convergente e da garganta, assim como ocorrido nos casos anteriores. Porém, para todas as vazões de líquido, a queda de pressão calculada pelo modelo não foi representada pelas simulações na secção divergente do equipamento. 
Figura 10 - Influência da vazão de líquido para injeção de gás constante $(\mathrm{G}-18120$ $\mathrm{m}^{3} / \mathrm{h}$ ), para o modelo de Viswanathan (1985)

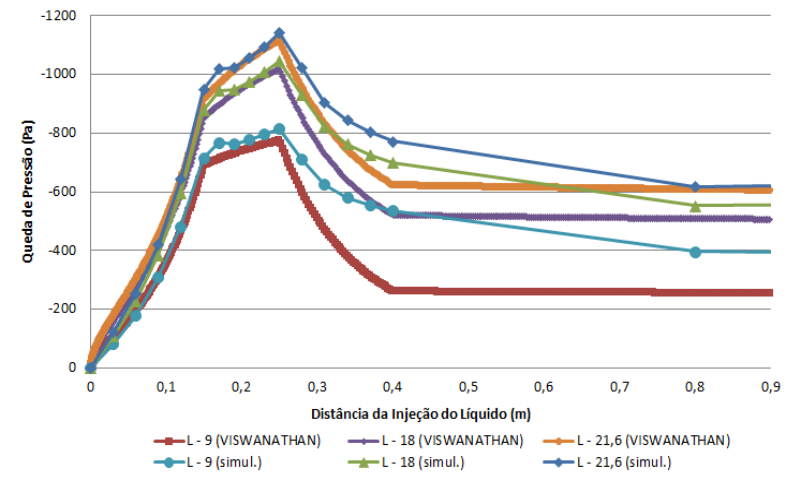

A Figura 11 mostra os valores das quedas de pressão média normalizadas para as diferentes simulações, para os planos distantes $0,8 \mathrm{~m}$ da entrada de líquido no lavador. A normalização da pressão foi feita agrupando-se as simulações com mesma vazão de líquido e dividindo-se o valor da queda de pressão pelo maior valor desta no grupo. Plotou-se esses valores em função da razão da vazão mássica de líquido pelo gás.

Pode-se notar que a maior queda de pressão para cada grupo ocorre quando a vazão da fase gás é a maior (diminuição da razão mássica).

Figura 11 - Quedas de pressão normalizadas pela razão mássica $(\mathrm{m})$ para a posição distante 0,8 metros da entrada de líquido

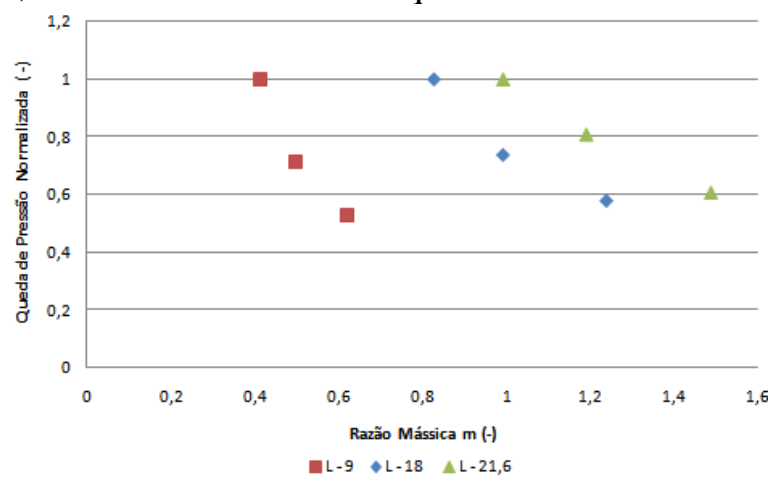

Para a condição de vazões do Item 2.1.1 plotaram-se a pressão (Figura 12), a velocidade da fase ar na direção do escoamento (Figura 13) e a fração de líquido
(Figura 14) para um plano YZ posicionado no centro do lavador. Pode-se perceber na Figura 12 o efeito do Venturi, o qual aumenta a velocidade do gás na região convergente (Figura 13) às custas da diminuição da pressão desse. Após a passagem do fluido pela garganta, nota-se a recuperação da pressão devido à diminuição da sua velocidade.

Na Figura 13 percebe-se uma zona de recirculação perto da parede do lavador na região divergente, possivelmente explicando o motivo das simulações divergirem dos modelos utilizados, que são unidirecionais, uma vez que esses consideram uma velocidade igual do fluido nas secções transversais do lavador, ou seja, não consideram em sua formulação regiões de recirculação.

Figura 12 - Pressão ao longo do lavador, para um plano YZ no centro desse.

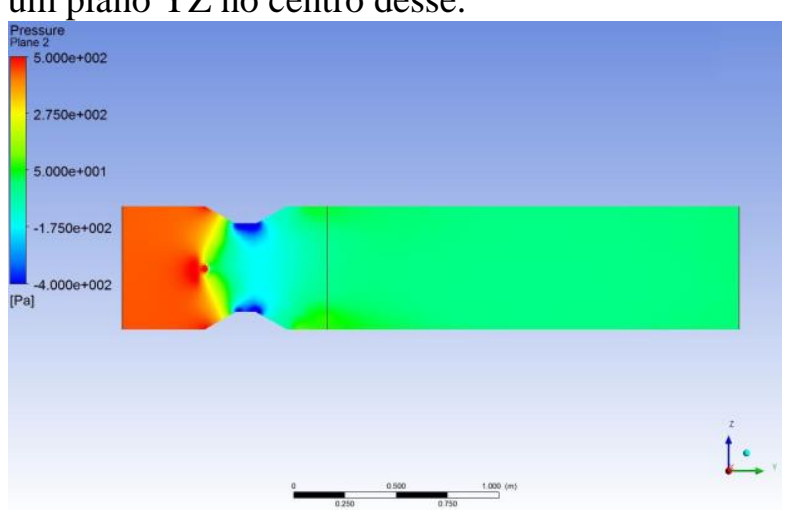

Figura 13 - Velocidade da fase contínua na direção $\mathrm{Y}$ ao longo do lavador, para um plano YZ no centro desse.

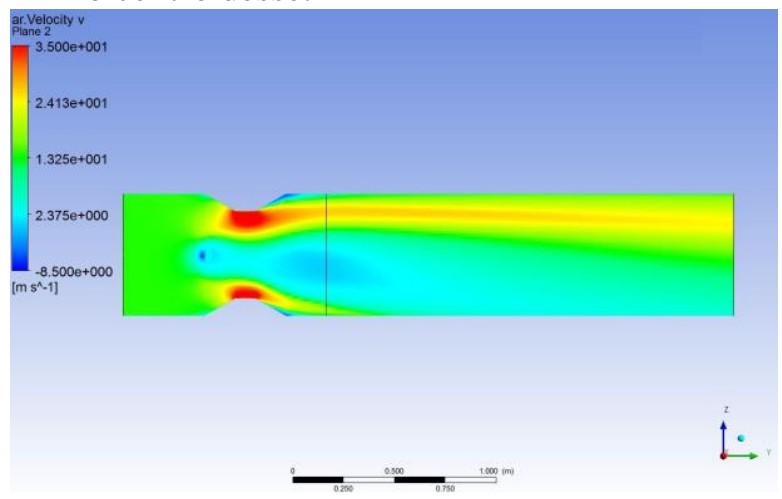


Pela Figura 14 percebe-se o efeito da força da gravidade adicionada nas simulações (sentido contrário ao eixo Z) e a não dispersão total da fase líquida ao longo do lavador, uma vez que admitiu-se velocidade nula para a fase líquida.

Figura 14 - Valores da fase líquida ao longo do lavador, para um plano $\mathrm{YZ}$ no centro desse.

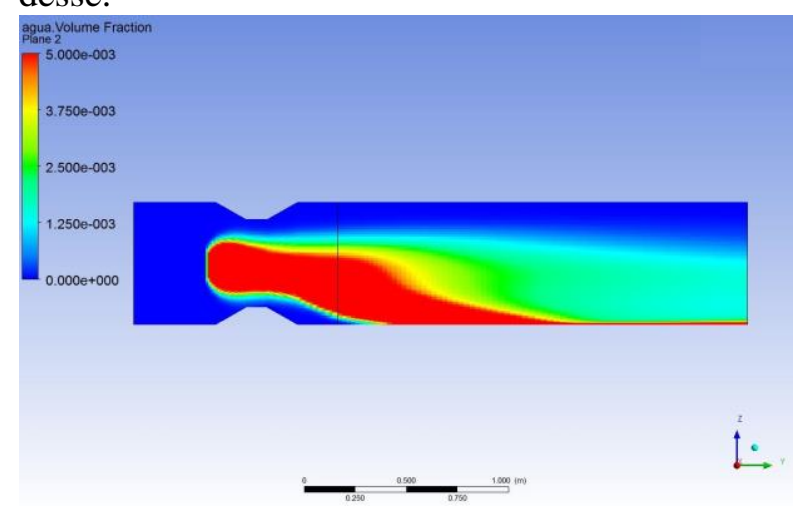

\section{CONCLUSÕES}

A simulação de um lavador Venturi industrial foi realizada para diferentes condições de vazões de líquido e gás, utilizando a abordagem Euler-Euler e o modelo de fluidos interpenetrantes para a resolução do escoamento bifásico. Compararam-se os resultados obtidos com os modelos clássicos da literatura, uma vez que não foi possível se coletar dados experimetais.

Observa-se que os resultados obtidos das simulações apresentam uma razoável concordância com os modelos clássicos na previsão da variação de pressão na região convergente e na garganta, porém apresentam divergências, em alguns casos, para a região divergente.

\section{NOMENCLATURA}

$a_{c}$ razão entre a área ocupada pela região central do escoamento e $\mathrm{A}_{\mathrm{T}}(-)$ $A_{T} \quad$ área tranversal total do equipamento $\left(\mathrm{m}^{2}\right)$
$D_{32}$

$D_{h}$

$f$

G

$L$

$m$

$\overline{M_{a b}}$

$M_{c}$

$P_{a}$

Re

$S_{a}$

$\frac{\overrightarrow{u_{a}}}{u_{b}}$

$v_{d}$

$v_{f}$

$v_{g}$

$v_{g g}$

$x$

$X_{a}$

$\alpha_{g}$

$\rho_{a}$

$\rho_{c}$

$\rho_{g}$

$\phi_{c}^{2}$

$\mu_{a}$ parâmetro de entrada de líquido no ecoamento central (-) coeficiente de arrasto (-) diâmetro de Sauter das gotas (m) diâmetro da secção do lavador (m) coeficiente de atrito de Darcy (-) vazão de gás com poluente $\left(\mathrm{m}^{3} \cdot \mathrm{s}^{-1}\right)$ vazão de líquido utilizada $\left(\mathrm{m}^{3} \cdot \mathrm{s}^{-1}\right)$ razão mássica entre líquido e gás (-) transferência de quantidade de movimento entre fases a e b $\left(\mathrm{kg} \cdot \mathrm{m}^{-2} \cdot \mathrm{s}^{-2}\right)$ vazão mássica do escoamento central $\left(\mathrm{kg} . \mathrm{s}^{-1}\right)$

pressão termodinâmica da fase a $(\mathrm{Pa})$ número de Reynolds (-) termo fonte de massa da fase $\left(\mathrm{kg} \cdot \mathrm{m}^{-3} \mathrm{~s}^{-1}\right)$ vetor de velocidade da fase a $\left(\mathrm{m} \cdot \mathrm{s}^{-1}\right)$ vetor de velocidade da fase $b\left(\mathrm{~m} . \mathrm{s}^{-1}\right)$ velocidade das gotas de líquido $\left(\mathrm{m} . \mathrm{s}^{-1}\right)$ velocidade do filme de líquido na parede $\left(\mathrm{m} \cdot \mathrm{s}^{-1}\right)$ velocidade do gás $\left(\mathrm{m} . \mathrm{s}^{-1}\right)$ velocidade do gás na garganta $\left(\mathrm{m} . \mathrm{s}^{-1}\right)$ comprimento do lavador $(\mathrm{m})$ fração volumétrica da fase a (-) razão volumétrica entre gás e líquido no escoamento central (-) densidade da fase a $\left(\mathrm{kg} \cdot \mathrm{m}^{-3}\right)$ densidade da região central do escoamento $\left(\mathrm{kg} \cdot \mathrm{m}^{-3}\right)$ densidade da fase gás $\left(\mathrm{kg} \cdot \mathrm{m}^{-3}\right)$ multiplicador para o gradiente de pressão em escoamento bifásico (-) viscosidade da fase a (Pa.s)

\section{REFERÊNCIAS}

ANSYS. CFX-Solver Theory Guide 15.0. ANSYS inc., 2013.

AHMADVAND, A.; TALAIE, M. R. CFD Modeling of Droplet Dispersion in a Venturi Scrubber. Chemical Engineering Journal, v.160, p.423-431, 2010. 
BOLL, R. H. Particle Collection and Pressure Drop in Venturi Scrubbers. Industrial and Engineering Chemicals Fundamentals, v.12, p.40-50, 1973.

BOLL, R. H.; FLAIS, L. R.; MAURER, P. W.; THOMPSON, W.L. Mean Drop Size in a Full Scale Venturi Scrubber via Transmissometer. Journal of the Air Pollution Control Association, v.24, p.934938, 1974.

GONÇALVES, J. A. S.; ALONSO, D. F.; COSTA, M. A. M.; AZZOPARDI B. J.; COURY J. R. Evaluation of the Models Available for the Prediction of Pressure Drop in Venturi Scrubbers. Journal of Hazardous Materials, v. B81, p.123-140, 2001.

GUERRA, V.G.; BÉTTEGA, R.; GONÇALVES, J. A.; COURY, J. R. Pressure Drop and Liquid Distribution in a Venturi Scrubber: Experimental Data and CFD Simulation. Industrial \& Engineering Chemistry Research, v. 51, p.8049-8060, 2012.

LEITH, D.; COOPER, D. W.; RUDNICK, S. N. Venturi Scrubbers: Pressure Loss and Regain. Aerosol Science Technology, v.4, p.239-243, 1985.

MAJID, A.; CHANGQI, Y.; ZHONGNING, S.; JIANJUN, W.; HAIFENG, G. CFD Simulation of Dust Particle Removal Efficiency of a Venturi Scrubber in CFX. Nuclear Engineering and Design, v.256, p.169-177, 2013.

PAK, S. I.; CHANG, K. S. Performance Estimation of a Venturi Scrubber Using a Computational Model for Capturing Dust Particles with Liquid Spray. Journal of Hazardous Materials, v.B138, p.560-573, 2006.
RAHIMI, A.; NIKSIAR, A.; MOBASHERI, M. Considering Roles of Heat and Mass Transfer for Increasing the Ability of Pressure Drop Models in Venturi Scrubbers. Chemical Engineering and Processing, v.50, p.104112, 2011.

VISWANATHAN, S. Annular Flow Pressure Drop Model for Pease-Anthony-Type Venturi Scrubbers. AIChE Journal, v.31, p.19471958, 1985.

YUNG, S.; BARBARIKA, H.F.; CALVERT, S. Pressure Loss in Venturi Scrubbers. Journal of the Air Pollution Control Association, v.27, p.348-351, 1977.

\section{AGRADECIMENTOS}

Os autores gostariam de agradecer à Agência fomentadora CAPES e à empresa ECOPLAS pelas informações e assistência oferecida. 